\title{
Skin and composite grafts
}

\author{
Katherine Hicks ${ }^{1}$, J. Regan Thomas ${ }^{2}$ \\ 1Department of Otolaryngology Head \& Neck Surgery, University of Texas Medical Branch, Galveston, TX 77019, USA. \\ 2Department of Otolaryngology Head \& Neck Surgery, Northwestern McGaw Medical Center, Chicago, IL 60611, USA. \\ Correspondence to: Dr. Katherine Hicks, Department of Otolaryngology Head \& Neck Surgery, University of Texas Medical \\ Branch, 301 University Blvd., Galveston, TX 77555-0521, USA. E-mail: kehicks@utmb.edu
}

How to cite this article: Hicks K, Thomas JR. Skin and composite grafts. Plast Aesthet Res 2022;9:2.

https://dx.doi.org/10.20517/2347-9264.2021.65

Received: 15 Jun 2021 First Decision: 26 Oct 2021 Revised: 22 Nov 2021 Accepted: 6 Dec 2021 Published: 14 Jan 2022

Academic Editor: Wen-Guo Cui Copy Editor: Yue-Yue Zhang Production Editor: Yue-Yue Zhang

\begin{abstract}
Skin grafts may be used for coverage of facial defects in situations in which alternative methods of reconstruction, such as local flaps, are not an option. They may also be beneficial for patients who wish to avoid or who are not good candidates for more complex reconstruction. Full-thickness skin grafts often have a better color and texture match to adjacent skin when compared to split-thickness grafts; however, split-thickness grafts have lower metabolic demand and increased survival rate. Composite grafts may be very useful in the repair of defects with unique contour and support requirements, such as the nasal ala and eyelid. With all grafts, thoughtful planning and sound surgical technique are critical in achieving the best possible functional and aesthetic result.
\end{abstract}

Keywords: Split-thickness skin grafts, full-thickness skin grafts, composite grafts, nasal reconstruction, eyelid reconstruction

Skin grafts may be used for coverage of facial defects in situations in which alternative methods of reconstruction, such as local flaps, are not an option. They may also be beneficial for patients who wish to avoid or who are not good candidates for more complex reconstruction. Full-thickness skin grafts often have a better color and texture match to adjacent skin when compared to split-thickness grafts; however, split-thickness grafts have lower metabolic demand and increased survival rate. Composite grafts may be very useful in the repair of defects with unique contour and support requirements, such as the nasal ala and eyelid. With all grafts, thoughtful planning and sound surgical technique are critical in achieving the best 
possible functional and aesthetic result.

Keywords: Split-thickness skin grafts, full-thickness skin grafts, composite grafts, nasal reconstruction, eyelid reconstruction

\section{INTRODUCTION}

Skin grafts and composite grafts have many uses in the repair of facial defects. They are well-suited for defects for which local or regional flaps are not viable options or for patients who are not interested in or medically stable enough for a more extensive procedure. Careful planning and precise execution of the plan during surgery are paramount to the success of these procedures. As always, it is important to have a comprehensive discussion with patients regarding all options for reconstruction prior to making a final decision. This discussion should include details regarding the procedure, post-operative care, and long-term expectations.

\section{SKIN GRAFTS}

\section{Principles}

Skin grafts are comprised of full-thickness epidermis and varying amounts of dermis, depending on the desired thickness of the graft. As demonstrated in Figure 1, a split-thickness skin graft contains epidermis and variable amounts of dermis, while a full-thickness graft contains full-thickness dermis in addition to full-thickness epidermis ${ }^{[1]}$.

Skin thickness varies by age, sex, and region of the body. A newborn's skin thickness is approximately 3.5 times thinner than an adult's at birth and nears adult thickness around five years of age ${ }^{[2]}$. In general, females have thinner skin than males. The thinnest skin on the body is found on the eyelids, while the thickest is on the palms of the hands and the soles of the feet ${ }^{[2]}$.

By definition, grafts are separated from their host blood supply and transferred to a recipient bed for coverage of a defect. Thus, when reconstructing defects with skin grafts, there are size and thickness constraints that should be adhered to in order to optimize the survival of the graft. There are three stages through which a graft progresses to obtain nutrients: (1) imbibition $(0-48 \mathrm{~h})$, in which nutrients are absorbed from plasma by capillary action; (2) inosculation (> 24-48 h), in which vascular buds from wound bed connect with preexisting vessels in graft; and (3) neovascularization $(>72 \mathrm{~h})$, in which the graft forms new vascular networks ${ }^{[3]}$.

\section{Uses}

On the face, skin grafts tend to have less optimal color and texture match when compared with local flaps. Split-thickness skin grafts, in particular, tend to appear atrophic and exhibit a shiny appearance, standing out in comparison with adjacent skin. They are also more prone to retraction and wound contracture if subjected to trauma or infection ${ }^{[4]}$. Full-thickness skin grafts have better color and texture match and are more resistant to local trauma, but they have a higher risk of failure due to higher metabolic demands on the graft as it is healing ${ }^{[4]}$.

In general, skin grafts may be considered for coverage of defects in the following instances: (1) healing by secondary intention would produce suboptimal results (ex., convex region of face); (2) coverage of large but thin defect is needed; (3) local flap coverage is not feasible; or (4) patient prefers to avoid more extensive procedure $^{[4]}$. 


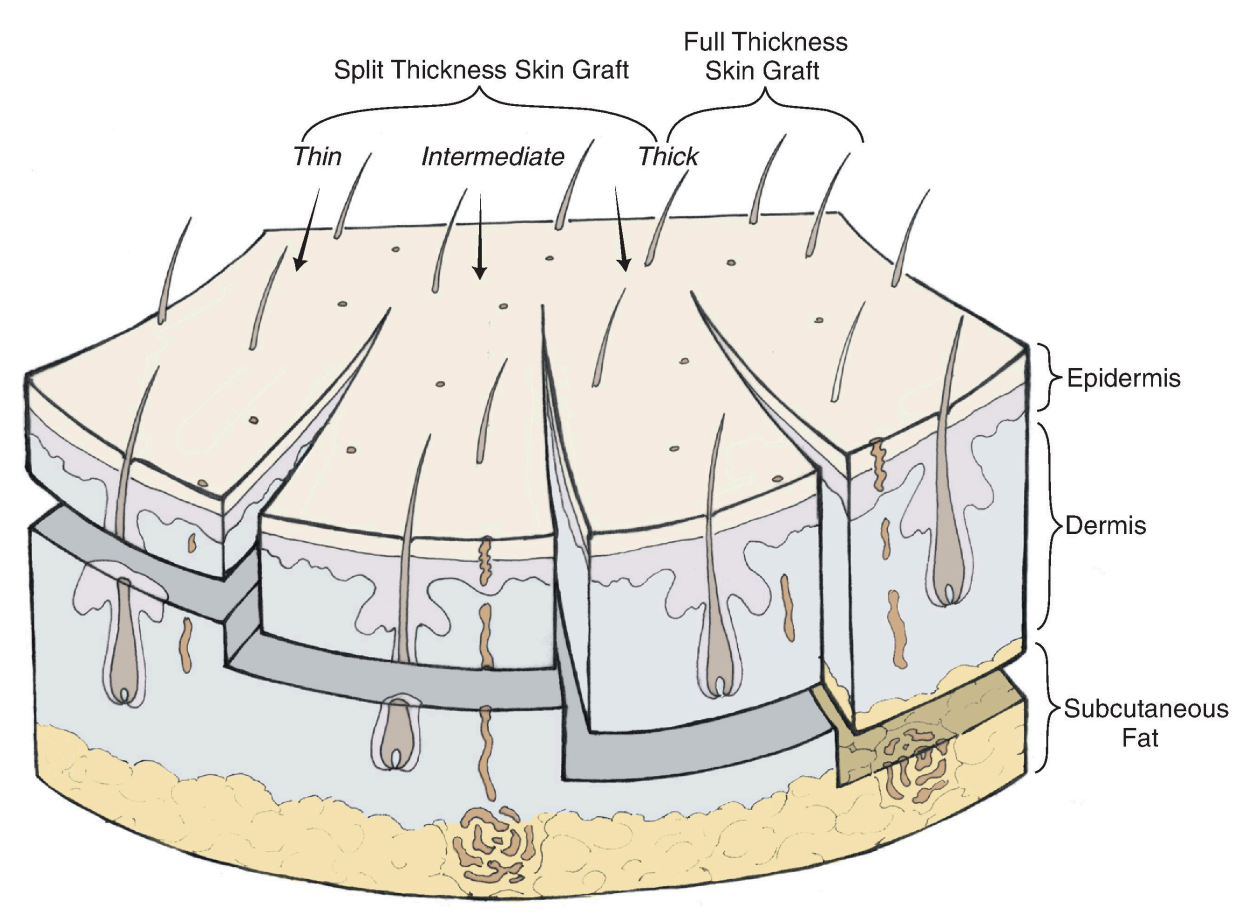

Figure 1. Thickness and content of various split- and full-thickness skin grafts. (Reused Figure 15-6 in Local Flaps in Facial Reconstruction, 3rd ed., with permission from Elsevier) ${ }^{[1]}$.

\section{Split-thickness skin grafts}

Split-thickness grafts range from 0.01 to 0.018 inches in thickness ${ }^{[4]}$. Because these grafts are thinner than full-thickness grafts, there is a shorter distance required for revascularization and a lower associated metabolic rate ${ }^{[4]}$. For these reasons, graft survival with split-thickness grafts is higher than with fullthickness grafts. In addition, these thin grafts do not contain hair follicles; thus, no hair will grow at the recipient site.

The primary advantage to using split-thickness skin grafts is the ability to cover a relatively large area with a high graft survival rate, as long as the recipient bed carries an adequate blood supply ${ }^{[4]}$. Placing a graft directly on the bone will result in graft failure. Areas that have been previously radiated have compromised blood supply, and skin grafts in these areas will have a lower survival rate. Split-thickness grafts may be harvested and applied in an expeditious manner, making them an attractive option to some patients who wish to avoid longer operative times and more involved procedures.

Disadvantages are primarily related to the appearance of split-thickness grafts. They often have a poor color and texture match to adjacent areas of the face. Compared to full-thickness grafts and local flaps, they exhibit increased contracture and decreased durability in cases of trauma or infection ${ }^{[4]}$.

The technique for harvesting and applying split-thickness grafts is straightforward, but grafts should be handled gently to avoid trauma. The donor region is prepped and draped; common donor sites include the thigh, buttocks, and abdominal wall. There are a few options for instrumentation, including an electrical dermatome, pneumatic dermatome, or manual option, such as a Humby knife. These authors prefer to use a pneumatic dermatome set to a thickness between 0.014-0.016 inches. The desired length of the graft is marked out on the donor site, and a dermatome blade of appropriate width is selected. A small amount of sterile mineral oil is applied to the blade and the patient's skin at the donor site. The dermatome is placed 
on the skin at a 45-degree angle, and firm downward pressure is placed. The instrument is turned on, dropped to an angle closer to parallel to the patient's skin, and slowly advanced forward while applying firm, even pressure. The instrument is turned off once a sufficiently long graft has been obtained, and the graft is removed from the dermatome and placed in sterile saline. A dressing is placed at the donor site to ensure that the site stays occluded and protected for about a week to optimize pain control and healing. Commonly, a hemostatic agent such as thrombin spray is applied prior to placing a dressing.

The recipient site is prepared, ensuring a "fresh" wound bed and adequate blood supply. A few small stab incisions may be made in the graft to allow egress of serous fluid after placement. The graft is sutured onto the recipient side, taking care to ensure that the epidermal side is facing superficially. Suturing in a "ship to shore" (first bite through the recipient bed, second through graft) method is usually best. The edges of the skin graft are trimmed as needed, taking care not to trim too much and produce tension on the graft. A bolster is sutured onto the recipient site. A common practice is to use interrupted sutures and leave some tails long, using these to tie over a bolster at the conclusion of the procedure. A variety of materials have been used for bolsters. This author prefers to use betadine-soaked cotton balls wrapped in xeroform dressing to optimize antimicrobial effects and moisture. The bolster is very important for healing and must be designed to provide gentle pressure to the wound, resulting in immobilization of the graft. If a suboptimal bolster is placed and the graft is allowed to move, shearing forces may result in hematoma or seroma formation and disrupt critical ingrowth of new blood vessels, compromising the survival of the graft. The bolster is removed approximately one week after surgery, and wound care with gentle cleansing and ointment application is initiated with the goal of keeping the graft clean and moist while it heals.

\section{Full-thickness skin grafts}

Full-thickness grafts, by definition, contain the entire epidermis and full-thickness dermis. It is important not to include subcutaneous fat in the grafts, as it acts as a barrier to vascularization ${ }^{[4]}$. They generally have a better color and texture match to adjacent skin on the face than split-thickness grafts. They also tend to have less atrophy and lack the "shiny" appearance that is characteristic of split-thickness grafts ${ }^{[4]}$. Areas of the face that are commonly reconstructed using full-thickness grafts are those with relatively thin skin and a paucity of adjacent skin available for local flaps, namely the nasal tip, eyelids, and ears. Figure 2 demonstrates a patient with a $1.5 \mathrm{~cm}$, partial thickness nasal tip defect. Given the small, thin nature of the defect and the patient's desire to avoid a more extensive procedure, the decision was made to proceed with a full-thickness graft to her nasal tip, harvested from the infraauricular skin.

Disadvantages of full-thickness grafts include slower healing rates and ultimately a lower survival rate, on average $^{[4]}$. In some cases, there may be increased donor site morbidity, although much of this risk is mitigated with proper donor site selection and sound surgical technique. These grafts do contain hair follicles and will grow hair at the recipient site, so this should be considered in graft selection ${ }^{[4]}$.

Common donor sites for full-thickness grafts in facial reconstruction include preauricular, postauricular, upper eyelid, nasolabial, and supraclavicular regions [Figure 3]. These sites have relatively thin skin, and incisions can be designed to be inconspicuous after healing. It is important to select a donor site whose skin closely matches the color and texture of the skin adjacent to the defect. A classic example of this is utilizing contralateral upper eyelid skin to repair an upper eyelid defect.

There are devices available for harvesting full-thickness grafts, but in the experience of these authors, fullthickness skin grafts are most commonly harvested using a scalpel. A template of the defect is created, often using foil from a suture packet. This template is moved to the donor area and placed in an orientation that 

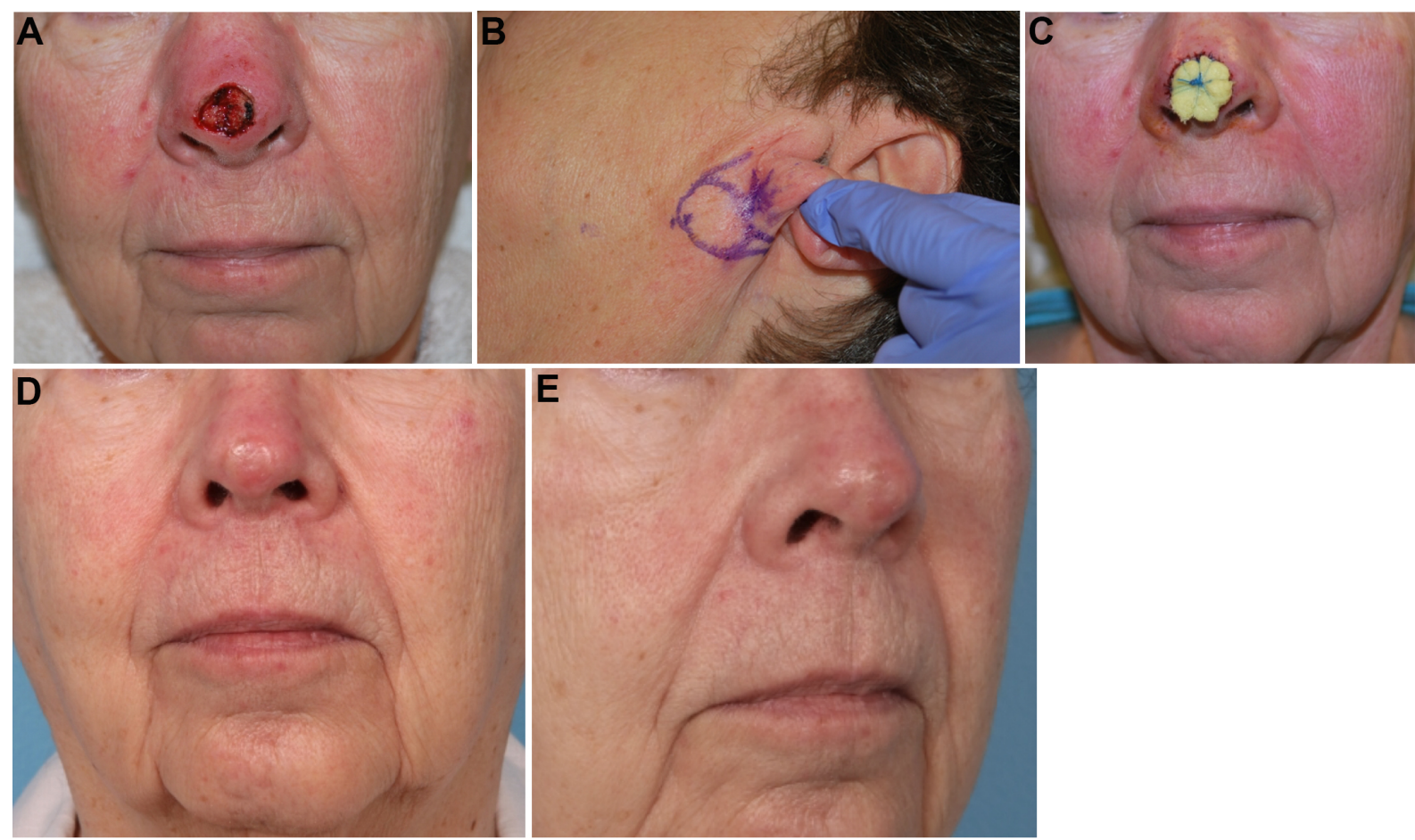

Figure 2. (A) Initial nasal tip defect. (B) Planned full-thickness skin graft. (C) Bolster in place, post-procedure. (D) and (E) frontal and oblique views 4 months post-procedure. [Second author's (J. Regan Thomas) patient].

facilitates favorable wound closure. Typically, an ellipse is marked around the template in an orientation parallel to or within resting skin tension lines. Incisions are made with a \#15 blade, and the graft is raised using a scalpel or sharp scissors. The graft is carefully de-fatted prior to placement in the recipient wound bed.

The donor site may be closed by undermining and closing primarily, or a split-thickness skin graft may be harvested from a second donor site for coverage. An example of utilizing this secondary skin graft may be found during microtia reconstruction; in cases in which there is insufficient skin to cover the anterior surface of the reconstructed ear, a full-thickness graft may be taken from the contralateral postauricular region. This site is difficult to close primarily; thus, a secondary skin graft may be harvested from the thigh or groin for coverage. Because the primary donor site is behind the ear, it is less critical to have a precise color and texture match.

The recipient site is prepared in the same manner as for a split-thickness graft, ensuring adequate vascularity. The full-thickness graft is placed and secured, ensuring that there is no tension across the graft. A gentle bolster is placed, applying firm pressure on the graft to prevent shearing.

\section{COMPOSITE GRAFTS}

\section{Principles}

Composite grafts contain two or more layers of tissue, most commonly skin, cartilage, and other tissue as indicated. They can provide a combination of coverage, contour, and support. However, due to the increased metabolic demand of these grafts, they are at a higher risk for failure than skin grafts ${ }^{[5]}$. To optimize perfusion, these grafts are traditionally designed to be no larger than $1 \mathrm{~cm}$ in any dimension to ensure that no portion of the graft is more than $5 \mathrm{~mm}$ from a vascularized wound edge ${ }^{[6]}$. The 


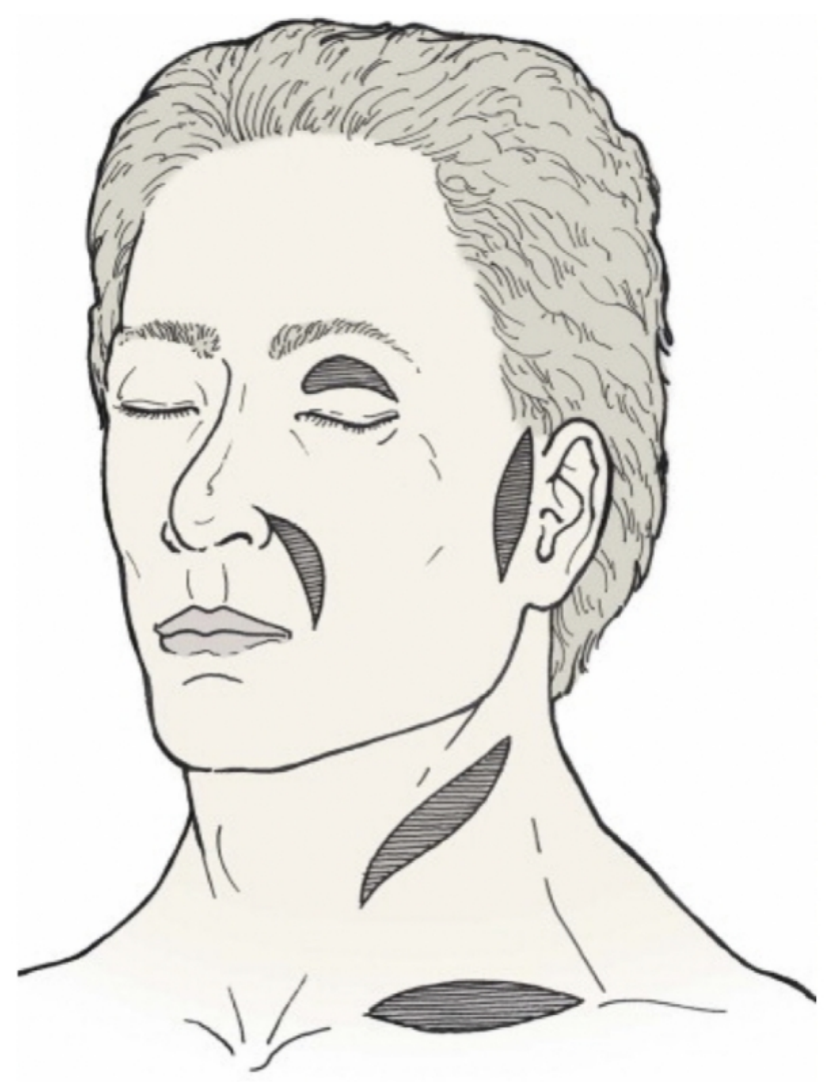

Figure 3. Common sites for harvesting full-thickness skin grafts for facial reconstruction. (Reused Figure 15-13 in Local Flaps in Facial Reconstruction, 3rd ed., with permission from Elsevier ${ }^{[1]}$.

vascularization process mimics that of skin grafts, although host vessels cannot penetrate cartilage ${ }^{[6]}$. Survival of composite grafts is contingent upon adequate revascularization. Careful surgical planning and meticulous technique help in this pursuit. Additionally, the following patient factors are recommended to optimize patient selection and survival of grafts: patient age $<65$ years, no tobacco use, and no systemic disease processes that would compromise revascularization ${ }^{[6]}$.

\section{Uses}

Composite grafts are typically used to repair small defects that require not only good color and texture match but also a degree of contouring and support. As mentioned above, typically, these grafts are kept to $1 \mathrm{~cm}$ or smaller in all dimensions. However, larger grafts may be used if they are being placed in a particularly vascular wound bed; constructing a hinge flap of vascular tissue is a technique that improves the exposure of graft to vascularized tissue ${ }^{[7]}$. For skin-cartilage composite grafts, harvesting a higher ratio of skin to cartilage results in graft behavior similar to that of a full-thickness skin graft, increasing the viability and enabling the use of a larger graft ${ }^{[6]}$. Composite grafts are frequently employed in repairing small, full- or partial-thickness defects of the eyelid, auricle, and nasal ala, columella, rim, and sill.

\section{Technique}

Harvesting and utilizing composite grafts is not technically difficult, but thoughtful planning is essential to the success of these repairs. An important consideration in the planning phase is that composite grafts tend to contract significantly, which can compromise the aesthetic and functional result if not accounted for. As such, these grafts may be intentionally oversized to compensate for contraction. 
The first step in repair is optimal preparation of the defect and ensuring optimal vascularization of wound bed. Baker advocates allowing the wound to heal by secondary intention first, followed by interval deepithelialization and recipient bed preparation in a second stage ${ }^{[6]}$. Other techniques, such as using a vascular hinge flap, may also be used to optimize the vascularity of the recipient bed.

Next, the donor site is selected, and the intended graft is marked out, oversizing slightly to allow for contracture. The graft is typically excised sharply, and judicious hemostasis is obtained. The donor site is closed in multiple layers.

The graft is contoured as needed and placed in the recipient bed. It is important to minimize the amount of cauterization used in this area. It is secured using the fewest number of strategically placed sutures possible, ensuring a secure repair while minimizing trauma to the delicate graft ${ }^{[6]}$. In general, the graft should fit well into the defect with no appreciable tension. After the graft has been sutured in place, a gentle bolster may be applied in select cases. If a bolster is not applied, the wound should be gently cleaned and kept moist with antibiotic ointment while healing. Sutures are typically removed one week after surgery. In most cases, minor revision of graft via contouring or dermabrasion may be done as early as eight to twelve weeks after the initial surgery.

\section{Composite grafts in nasal reconstruction}

The earliest description of composite grafts in nasal reconstruction was published by Konig ${ }^{[8]}$ in 1914, who used composite auricular grafts to repair defects of the nasal ala and reported a graft survival rate of $53 \%$. Since that time, understanding of graft physiology has improved, leading to a significantly higher graft survival rate.

Due to the unique shape and construction of the auricle, composite grafts from different parts of the auricle may be utilized to reconstruct various nasal defects [Figure 4$]^{[1]}$. For defects of the alar rim, the optimal graft varies based on where the defect is and what tissue layers are involved. Figure 5 demonstrates a patient with a small, full-thickness defect of the nasal ala, which was repaired with a hinge flap for the inner lining and a composite graft from the root of the helix for structural support and cutaneous coverage. For defects that are more lateral and contain mostly fibrofatty tissue, grafts from the lobule may be appropriate. Grafts from the tragus and anti-tragus may be useful in covering shallow defects along the alar margin. Defects involving the columella and soft tissue facet are often repaired with grafts from the helical root; these grafts have suitably thin skin, and adjacent preauricular skin may be harvested if necessary. For defects of the nasal sill or in the correction of vestibular stenosis, a sturdy graft is needed; a composite graft from the triangular fossa is well-suited for this area ${ }^{[6]}$.

In larger defects, in which a local flap is used in addition to a composite graft, it is beneficial to do the local flap first, allow this to heal, and then perform the composite graft as a second stage procedure ${ }^{[6]}$. This practice minimizes the tension on the graft as it is healing. This method may be used in combined cheek, lip, and nasal defects, in which a local flap is utilized to recreate the alar-facial or nasofacial junction. It is important to "set" this boundary first, so that it is well-defined and stable prior to the placement of the composite graft.

\section{Composite grafts in eyelid reconstruction}

In general, eyelid reconstruction is very complex, owing to the intricate aesthetic goals and functional requirements of the eyelid. As with all reconstructions, the eyelid must be thought of in layers: anterior lamella (skin and orbicularis muscle), septum, and posterior lamella (tarsal plate and conjunctiva). 


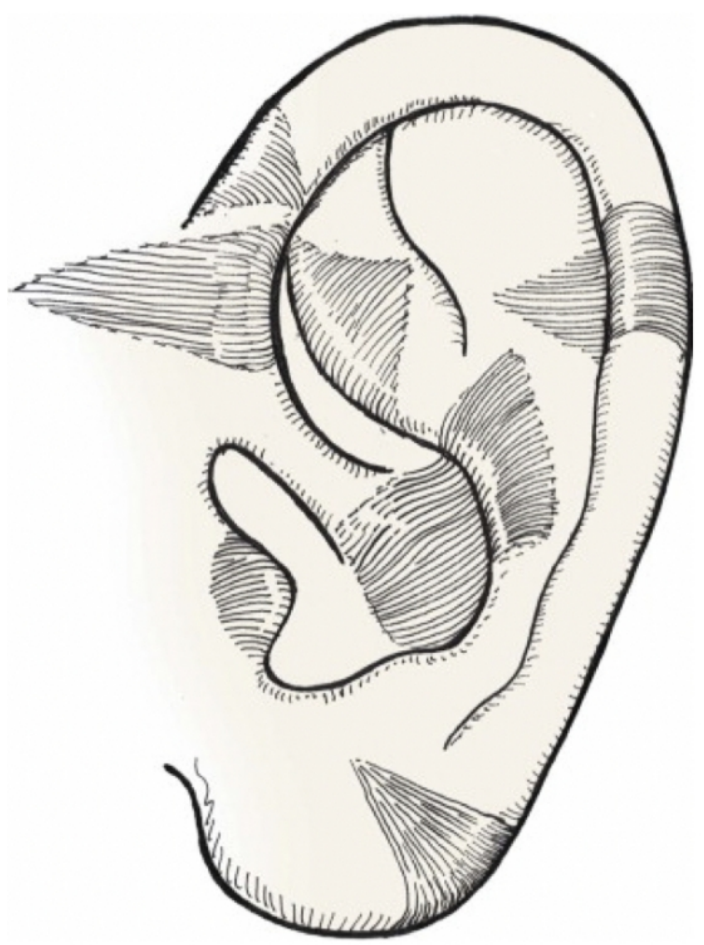

Figure 4. Common sites for the harvest of auricular composite grafts. (Reused Figure 15-23 in Local Flaps in Facial Reconstruction, 3rd ed., with permission from Elsevier) ${ }^{[1]}$.
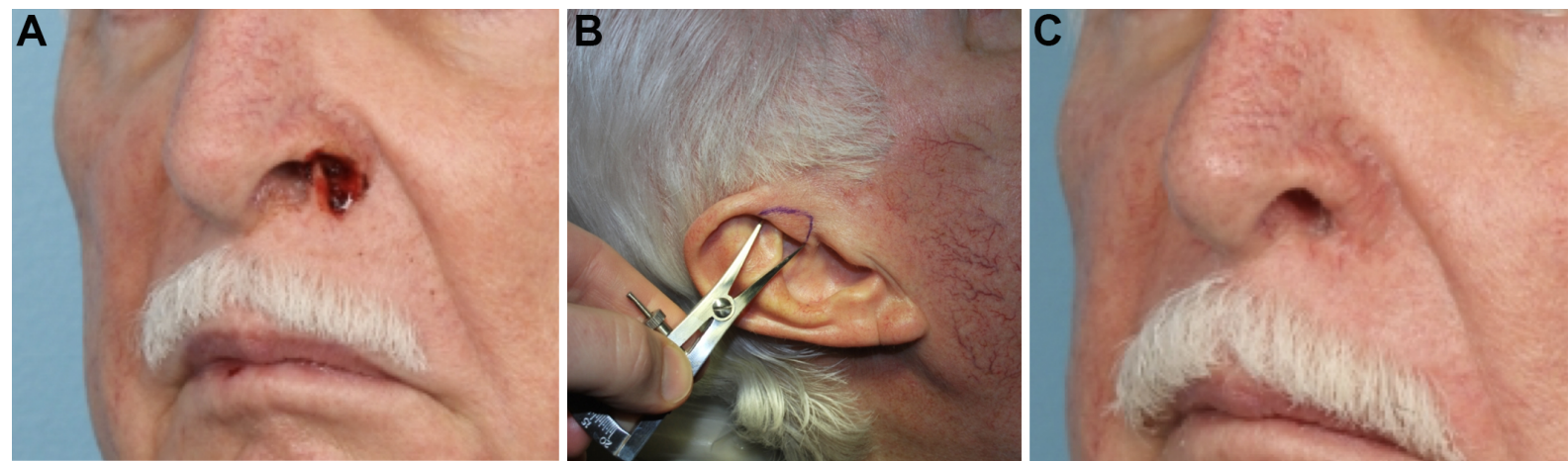

Figure 5. (A) Preoperative full-thickness defect of nasal ala. (B) Surgical plan for composite skin-cartilage graft from the root of helix. (C) Post-operative appearance 3 months later. (Courtesy of Dr. Douglas Sidle, MD, the photo is one of his patients).

Composite grafts of the eyelid are most commonly employed when a portion of the tarsus must be replaced $^{\left[{ }^{[}\right]}$. A multitude of local flaps and grafts have been described for the repair of eyelid defects, and there are often multiple options for the closure of a given defect. However, it is important to remember that grafts do not carry their own blood supply and should only be utilized by themselves (for small defects) or in conjunction with a local flap; they should never be used concurrently with another graft. For example, in a patient with a large, full-thickness lower eyelid defect, a composite graft may be selected to reconstruct the posterior lamella; in this case, a local flap must be used to cover the anterior lamella. In contrast, if a tarsoconjunctival flap were chosen for posterior lamellar reconstruction, then a skin graft could be used for anterior lamellar coverage. 
In contrast with most other grafts, composite grafts used in eyelid reconstruction are designed to be under some tension, such that adequate apposition of the eyelid to the globe is obtained postoperatively. Thus, it may be necessary to use supportive sutures or bolsters to avoid excessive tension while the graft is healing. A secondary canthoplasty with tarsal strip may ultimately be required for optimal results. During the closure, accurate reapproximation of the tarsal plate and eversion of the eyelid margin are critical steps in avoiding an irregular or notched appearance postoperatively.

First described by Callahan ${ }^{[10]}$ in 1951, one use of composite grafts for reconstruction of full-thickness eyelid defects is the utilization of a full-thickness pentagon-shaped graft from the contralateral lid $^{[9]}$. Depending on eyelid laxity and size of the graft, the donor defect may be closed primarily, or a lateral canthotomy may be done to facilitate closure. In these authors' practices, the use of such grafts is uncommon, as primary closure often works well for small defects, and full-thickness rotation-advancement flaps work well for larger defects and avoid donor site morbidity in the contralateral eyelid.

For the purpose of posterior lamellar reconstruction only, a free tarsoconjunctival graft may be harvested from the upper lid. The tarsal plate is wider in the upper lid, so a graft may be taken from here, as long as care is taken not to traumatize the upper eyelid retractors. In this case, a local flap would be utilized for anterior lamellar coverage. A septal mucochondral composite graft may be used in a similar manner. Utilization of this graft plus a musculocutaneous flap from the upper eyelid has been described ${ }^{[11]}$.

Many reconstructive techniques for the eyelid result in a segment of the eyelid margin that lacks eyelashes, which may be extremely bothersome to some patients. The use of a thin graft harvested from the eyebrow and implanted along the eyelid margin has been described ${ }^{[12]}$. Eyelashes are oriented parallel to one another, are limited in length, and taper at their ends; eyebrow hairs are similar and are thus a logical choice for replacement. The rich vascular supply of the eyelid helps ensure graft survival. The brows must be closely analyzed to select the optimal donor site; this is often a diagonal section near the central portion of the ipsilateral brow. The graft is approximately $3 \mathrm{~mm}$ in width. Meticulous incision and suturing techniques must be used to minimize trauma to hair follicles.

\section{SKIN AND DERMAL SUBSTITUTES}

In some instances, the use of skin substitutes may be an appropriate option for or adjunct to the treatment of wound defects. Increased research and understanding in the fields of molecular biology and tissue engineering have led to the development of multiple skin substitute materials for use in wound care and reconstructive procedures ${ }^{[13]}$. Wound healing is a complex process involving many cell types, growth factors, and extracellular matrix components. In chronically inflamed or non-healing wounds, the inflammatory process inherent to the wound results in degradation of these components and prevention of normal healing. Skin substitutes essentially act as a scaffold, providing various combinations of cells, growth factors, and extracellular matrix components, depending on the specific product, with the ultimate goal of facilitating revascularization and reepithelialization of a given wound $\mathrm{d}^{[14,15]}$.

Perhaps the primary advantage of these products is the lack of donor site morbidity. Another relevant advantage is the potential for utilization of a large amount of material, as the availability is not limited by traditional donor site restrictions ${ }^{[1,15]}$. Conversely, there are challenges associated with product development and refinement, including the need for substantial research and investment in biocompatibility and application-specific optimization. In parallel with these development challenges lies the primary barrier to widespread adoption: high cost. At this time, there is limited availability of most of these products outside of large hospital systems $\mathrm{s}^{[14,15]}$. 
Skin substitutes may be utilized in various capacities, depending on the molecular composition and physical properties of a particular product ${ }^{[14,15]}$. Commonly described uses include the utilization of these products in the management of burns and chronic wounds, including chronic venous ulcers, diabetic foot ulcers, and pressure ulcers. One other application that is particularly relevant to the topic of skin and composite grafts is the utilization of a dermal substrate during two-stage skin cancer resection and reconstruction. A recent study evaluated the use of Hyalomatrix in 45 medically fragile patients with non-melanoma skin cancer ${ }^{[16]}$. These patients underwent excision of their cancer and application of Hyalomatrix in the first stage, followed by application of skin graft or healing by secondary intention (if patient preference or patient found to be unstable for further surgery). The use of the matrix induces neodermis formation with the goal of optimizing skin graft take. Matrix was left in place for a mean duration of 19 days, and subsequent skin graft placement demonstrated $95 \%-100 \%$ take. Of note, the reepithelialization time was relatively similar with and without skin grafting, taking 31 days $v s .46$ days, respectively. However, patient satisfaction scores were significantly higher following skin graft placement ${ }^{[16]}$.

As the development of these products continues to advance, there will be increased potential for their utilization in the management of wounds. Therefore, it is important that clinicians stay abreast of these advances and consider the incorporation of these products into their treatment algorithms when appropriate.

\section{CONCLUSIONS}

Split-thickness skin grafts, full-thickness skin grafts, and composite grafts have many uses in facial reconstruction, particularly in patients who wish to avoid a more extensive procedure. Thoughtful surgical planning and meticulous technique are equally important in achieving optimal outcomes. An honest and thorough discussion of all surgical options and anticipated results should be conducted with patients prior to undergoing reconstructive procedures.

\section{DECLARATIONS}

\section{Authors' contributions}

Made substantial contributions to researching and reviewing publications and compiling manuscript: Hicks K

Made substantial contributions through provision of resources, organization of topic, and guidance throughout writing: Thomas JR

\section{Availability of data and materials}

Not applicable.

\section{Financial support and sponsorship}

None.

\section{Conflicts of interest}

Both authors declared that there are no conflicts of interest.

\section{Ethical approval and consent to participate}

Not applicable.

\section{Consent for publication}

Figures are obtained copyright permission from Elsevier. The authors state that the photos to be identified don't need to be obtained consent from patients. 


\section{Copyright}

(c) The Author(s) 2022.

\section{REFERENCES}

1. Jewett B. Skin and composite grafts. In: Baker S, editor. Local flaps in facial reconstruction. 3rd ed. Saunders; 2014. p. 339-67.

2. Southwood WF. The thickness of the skin. Plast Reconstr Surg (1946) 1955;15:423-9. DOI

3. MIR y MIR L. Biology of the skin graft; new aspects to consider in its revascularization. Plast Reconstr Surg (1946) 1951;8:378-89. DOI PubMed

4. Hom D, Tope W, Murakami C. Minimally invasive options and principles for cutaneous reconstruction. In: Papel I, Frodel J, Holt R, Nachlas N, Park S, editors. Facial plastic and reconstructive surgery. 4th ed. Thieme Medical Publishers; 2016. p. 600-19.

5. Konior R. Free composite grafts. Otolaryngol Clin North Am 1994;27:81. PubMed

6. Jewett B, Baker S. Skin and composite grafts. In: Baker S, editor. Principles of nasal reconstruction. Springer; 2011. p.133-61.

7. Converse J. Reconstructive plastic surgery, vol. 2. Philadelphia, PA: WB Saunders; 1964.

8. Konig F. auUber nasenplastik. Beitr Klin Chir 1914;94:515. DOI

9. Cannon PS, Madge SN, Kakizaki H, Selva D. Composite grafts in eyelid reconstruction: the complications and outcomes. $\mathrm{Br} J$ Ophthalmol 2011;95:1268-71. DOI PubMed

10. Callahan A. The free composite lid graft. AMA Arch Ophthalmol 1951;45:539-45. DOI PubMed

11. Bae Y, Kim S, Hwang S, Kim K, Oh C. Reconstruction of the lower eyelid with septal mucochondral composite graft and upper eyelid musculocutaneous flap. J Korean Soc Plast Reconstr Surg 2004;31:309-314. DOI

12. Kasai K. Eyelash reconstruction with strip composite eyebrow graft. Ann Plast Surg 2008;60:649-51. DOI PubMed

13. Nyame TT, Chiang HA, Orgill DP. Clinical applications of skin substitutes. Surg Clin North Am 2014;94:839-50. DOI PubMed

14. Tenenhaus M, Rennekampff HO. Current concepts in tissue engineering: skin and wound. Plast Reconstr Surg 2016;138:42S-50S. DOI PubMed

15. Hughes OB, Rakosi A, Macquhae F, Herskovitz I, Fox JD, Kirsner RS. A review of cellular and acellular matrix products: indications, techniques, and outcomes. Plast Reconstr Surg 2016;138:138S-47S. DOI PubMed

16. Marcasciano M, Mazzocchi M, Kaciulyte J, et al. Skin cancers and dermal substitutes: is it safe? Review of the literature and presentation of a 2 -stage surgical protocol for the treatment of non-melanoma skin cancers of the head in fragile patients. Int Wound $J$ 2018;15:756-68. DOI PubMed PMC 